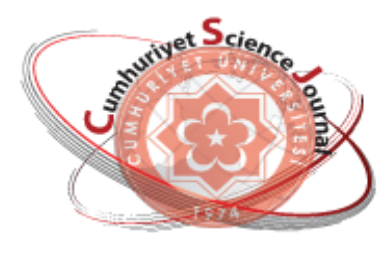

e-ISSN: $2587-246 X$

ISSN: $2587-2680$
Cumanoriyot Science Journal

Cost

Cumhuriyet Sci. J., Vol.38-3 (2017) 435-443

\title{
Effects of Quartic Wave-Vectors on Continuum Model of Phosphorene
}

\author{
Aybey MOGULKOC* \\ Department of Physics, Faculty of Sciences, Ankara University, 06100, Tandogan, Ankara, TURKEY \\ Received: 06.06.2017; Accepted: 19.07.2017 \\ http://dx.doi.org/10.17776/csj.340476
}

\begin{abstract}
Phosphorene is the monolayer structures of black phosphorous. Its low-energy physics can be described by continuum model in the scheme of $k \cdot p$ theory. Validity of energy range for this model can be improved by extra terms as trigonal warp effect in graphene. We construct a new continuum model by expanding the tight-binding Hamiltonian of phosphorene up to the order of $\mathrm{k}^{4}$. The new quartic model increases the energy range where the eigenvalues of continuum and tight-binding Hamiltonian are matched. Moreover, we examine the Landau levels of phosphorene by the influence of quartic terms. While quartic terms have insignificant effects on the lower Landau levels of phosphorene, they have non-negligible contributions to the higher levels. In this study, we propose a new continuum model which brings better description to the dynamics of phosphorene charge carriers.
\end{abstract}

Keywords: Phosphorene, continuum model, Landau levels

\section{Fosforenin Sürekli Modeli Üzerine Dördüncü Mertebeden Dalga Vektörünün Etkisi}

Özet: Fosforen siyah fosforun tek tabakalı yapısıdır. Düşük enerji fiziği k·p teorisi çerçevesinde sürekli model ile tasvir edilebilir. Sürekli modelin geçerli olduğu enerji aralığ grafendeki üçgensel eğrilikte olduğu gibi ek terimler ile artırılabilir. Burada fosforenin sıkı-bağ Hamiltonyeni $\mathrm{k}^{4}$ mertebesine kadar seri açılarak yeni bir sürekli model oluşturulmuştur. Dördüncü mertebeden yeni model sürekli ve sıkı-bağ Hamiltonyenlerinin özdeğerlerinin örtüştüğü enerji aralığını artırmıştır. Dahası dördüncü mertebeden terimin katkısı göz önünde bulundurularak fosforenin Landau seviyeleri incelenmiştir. Dördüncü mertebeden terimin düşük Landau seviyeleri üzerinde göz ardı edilebilir etkiye sahipken, yüksek Landau seviyelerine ihmal edilemeyen katkılar getirir. Bu çalışmada fosforenin yük taşıyıcılarının dinamiğini daha iyi tasvir eden yeni bir sürekli model önerilmiştir.

Anahtar Kelimeler: Fosforen, sürekli model, Landau seviyeleri

\section{INTRODUCTION}

With the discovery of graphene [1], twodimensional structures have become one of the active research area for the condensed matter physicist. Even graphene shows promising physical properties [2], its gapless electronic structure limits the technological application of this fascinating material. Due to the lack of band gap in graphene, researchers have been focused on the other two-dimensional (2D) structures, i.e., silicene, germanene, stanene. Recently, with the synthesis of a few-layer black phosphorus by the several methods including 
chemical vapor deposition [3], mechanical and liquid exfoliation [4-7], interest on this new material has been increased extensively related with its unique physical properties, i.e., relatively higher charge carrier mobility [6-8], tunable band gap with the number of layer $[9,10]$, and structural anisotropy [10-12]. While the monolayer black phosphorus (namely phosphorene) has a direct band gap of $1.5-2 \mathrm{eV}$ at the $\Gamma$ point of the Brillouin zone $[6,13,14]$, band gap of black phosphorus can be tuned by the number of layer between 0.3-2.0 eV [9.10]. Moreover, the phonon spectrum based on the density functional theory ensures the thermal stability of phosphorene [15].

To understand the band structure and charge carrier dynamics of the materials, several theoretical approaches have been developed such as density functional theory, tight-binding model and continuum model. Among these approaches, continuum model (or $k \cdot p$ model) is an important tool which perfectly describes the low-energy physics of two-dimensional structures. In graphene, the low-energy spectrum was constructed by the Dirac Hamiltonian [16] which also describes some relativistic phenomenas in graphene, i.e., Klein paradox [217]. The conic spectrum of graphene arising from Dirac Hamiltonian is valid only for the low energy region of band structure which describes the isotropic electronic properties of the charge carriers. However, by considering trigonal warp effect [2,18] which extends the continuum approach to the higher energies, the band structure becomes non-linear which yields some anisotropic properties in graphene $[19,20]$. Even the continuum model of phosphorene was constructed by considering the quadratic wavevectors [21,22], the contributions of higher order wave-vectors that increase the validity of continuum approach for the higher energies have never been discussed before.

In this paper, we construct a new continuum model by considering quartic terms $\left(k^{4}\right)$ in tightbinding expansion around $\Gamma$ point. The theory part of the paper consists of two sections as, A. Continuum model with quartic correction and B. Landau levels with quartic correction. At the first section, we formulate the quartic continuum Hamiltonian and find the energy eigenvalues of the system. In the presence of perpendicular magnetic field, Landau levels of phosphorene are examined by considering the quartic correction in Sec. B.

\section{THEORY}

\section{A. Continuum model with quartic correction}

The energy spectrum of phsophorene can be formulated by a four-band tight-binding model $[13,14,23]$. Moreover, $C_{2 h}$ group invariance of the phosphorene enables us to describe the system by two-band model [21-23]. The lowenergy physics of phosphorene can be described by $k \cdot p$ model in the long wave-length limit. It can be extracted by expanding the tightbinding Hamiltonian around $\Gamma$ point. In the framework quadratic wavevectors, the continuum Hamiltonian shows good agreement with the tight-binding model between -2.0 to $1.5 \mathrm{eV}[21,22]$. Moreover, the continuum Hamiltonian of phosphorene can be constructed by considering $k^{4}$ terms at the expansion of tight-binding Hamiltonian around $\Gamma$ point. In the long-wavelength limit, the quadratic continuum Hamiltonian of MBP can be written as [21],

$$
\mathcal{H}_{0}=\left[\begin{array}{cc}
u_{0}+\bar{\eta}_{x} p_{x}^{2}+\bar{\eta}_{y} p_{y}^{2} & \delta+\bar{\gamma}_{x} p_{x}^{2}+\bar{\gamma}_{y} p_{y}^{2}+i \bar{\chi} p_{y} \\
\delta+\bar{\gamma}_{x} p_{x}^{2}+\bar{\gamma}_{y} p_{y}^{2}-i \bar{\chi} p_{y} & u_{0}+\bar{\eta}_{x} p_{x}^{2}+\bar{\eta}_{y} p_{y}^{2}
\end{array}\right]
$$

By considering the tight-binding description of phosphorene in Ref. 21, we can expand the tight-binding Hamiltonian up to the $p^{4}$ terms, and the total Hamiltonian becomes, 


$$
\mathcal{H}_{0}=\left[\begin{array}{cc}
u_{0}+\bar{\eta}_{x} p_{x}^{2}+\bar{\eta}_{y} p_{y}^{2}+\bar{\tau}_{x} p_{x}^{4}+\bar{\tau}_{y} p_{y}^{4} & \delta+\bar{\gamma}_{x} p_{x}^{2}+\bar{\gamma}_{y} p_{y}^{2}+\bar{\kappa}_{x} p_{x}^{4}+\bar{\kappa}_{y} p_{y}^{4}+i \bar{\chi} p_{y} \\
\delta+\bar{\gamma}_{x} p_{x}^{2}+\bar{\gamma}_{y} p_{y}^{2}+\bar{\kappa}_{x} p_{x}^{4}+\bar{\kappa}_{y} p_{y}^{4}-i \bar{\chi} p_{y} & u_{0}+\bar{\eta}_{x} p_{x}^{2}+\bar{\eta}_{y} p_{y}^{2}+\bar{\tau}_{x} p_{x}^{4}+\bar{\tau}_{y} p_{y}^{4}
\end{array}\right]
$$

Here, $\bar{\eta}_{i}=\eta_{i} / \hbar^{2}\left(\eta_{x}=0.58 \mathrm{eV} \AA^{2}\right.$ and $\eta_{y}=$ $\left.1.01 \mathrm{eV} \AA^{2}\right), \bar{\gamma}_{i}=\gamma_{i} / \hbar^{2}\left(\gamma_{x}=3.93 \mathrm{eV} \AA^{2}\right.$ and $\left.\gamma_{y}=3.83 \mathrm{eV} \AA^{2}\right), \quad \bar{\chi}=\chi / \hbar\left(\chi=5.25 \mathrm{eV} \AA^{2}\right)$, $\bar{\tau}_{i}=\tau_{i} / \hbar^{4} \quad\left(\tau_{x}=-0.13 \mathrm{eV} \AA^{4} \quad\right.$ and $\quad \tau_{y}=$ $\left.-0.40 \mathrm{eV} \AA^{4}\right), \quad \bar{\kappa}_{i}=\kappa_{i} / \hbar^{4} \quad\left(\kappa_{x}=-0.89 \mathrm{eV} \AA^{4}\right.$ and $\left.\kappa_{y}=-2.08 \mathrm{eV} \AA^{4}\right), u_{0}=-0.42 \mathrm{eV}$ and $\delta=0.76 \mathrm{eV}$. The corresponding eigenvalues of total Hamiltonian in Eq.(2) can be found

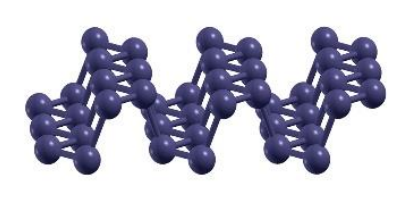

(a) side view

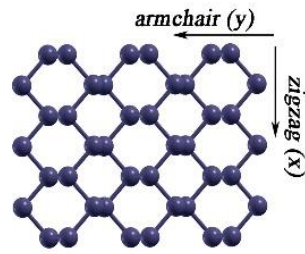

(b) top view

Figure 1. Puckered structure of phosphorene, (a) side view and (b) top view.

as,

$$
E_{\lambda}^{\text {quartic }}=u_{0}+\sum_{i=x, y}\left(\eta_{i} k_{i}^{2}+\tau_{i} k_{i}^{4}\right)+\lambda\left[\left(\delta+\sum_{i=x, y} \gamma_{i} k_{i}^{2}+\kappa_{i} k_{i}^{4}\right)^{2}+\left(\chi k_{y}\right)^{2}\right]^{1 / 2} .
$$

In absence of quartic terms, energy eigenvalues of Hamiltonian in Eq.(1) yield well-known quadratic expression in Ref. 21, i.e.

$$
E_{\lambda}^{\text {quadratic }}=u_{0}+\sum_{i=x, y} \eta_{i} k_{i}^{2}+\lambda\left[\left(\delta+\sum_{i=x, y} \gamma_{i} k_{i}^{2}\right)^{2}+\left(\chi k_{y}\right)^{2}\right]^{1 / 2} .
$$

Furthermore, square root term in Eq.(3) can be expanded for the small values of wave-vector which gives,

$$
\begin{aligned}
E_{\lambda}^{\text {quartic }} & \approx\left(u_{0}+\lambda \delta\right)+\left(\eta_{x}+\lambda \gamma_{x}\right) k_{x}^{2}+\left(\eta_{y}+\lambda \gamma_{y}+\lambda \frac{\chi^{2}}{2 \delta}\right) k_{y}^{2} \\
& +\left(\tau_{x}+\lambda \kappa_{x}\right) k_{x}^{4}+\left(\tau_{y}+\lambda \kappa_{y}-\lambda \frac{\gamma_{y} \chi^{2}}{2 \delta^{2}}-\lambda \frac{\chi^{4}}{8 \delta^{3}}\right) k_{y}^{4} .
\end{aligned}
$$

Here the analytical expression in Eq.(4) is valid only the vicinity of $\Gamma$ point. The coefficients $\left(\tau_{x}+\lambda \kappa_{x}\right.$ and $\tau_{y}+\lambda \kappa_{y}-\lambda \gamma_{y} \chi^{2} / 2 \delta^{2}-$ $\lambda \chi^{4} / 8 \delta^{3}$ ) of $k^{4}$ terms determine the contribution of quartic terms to the eigenvalues of phosphorene. While it takes $-1.03 \mathrm{eV} \AA^{4}(0.76$ $\AA^{4}$ ) along the zigzag direction, it becomes $-310.19 \mathrm{eV} \AA^{4}\left(309.4 \mathrm{eV} \AA^{4}\right)$ along the armchair direction for electrons (holes). It is easy to claim that the effect of $k^{4}$ term is more pronounced along the armchair direction for the low-energy spectrum of phosphorene. In the framework of quadratic continuum model, Hamiltonian in Eq.(1) have Schrodinger-like and Dirac-like character related with quadratic and linear terms, respectively, which is shown at Fig.2(a). As it is seen, linear term has non-negligible effect 
along the armchair direction. Moreover, it shows good agreement with tight-binding results between -2.0 to $1.5 \mathrm{eV}$. By inclusion of quartic terms, the range of overlap with tight-binding spectrum is increased up to $3.0 \mathrm{eV}(-3.0 \mathrm{eV})$ for conduction (valance) band. It can be easily claimed that the quartic continuum model describe the low-energy physics better than the quadratic model for phosphorene. Moreover, density of states (DOS) per unit-cell can be calculated by the following formula,

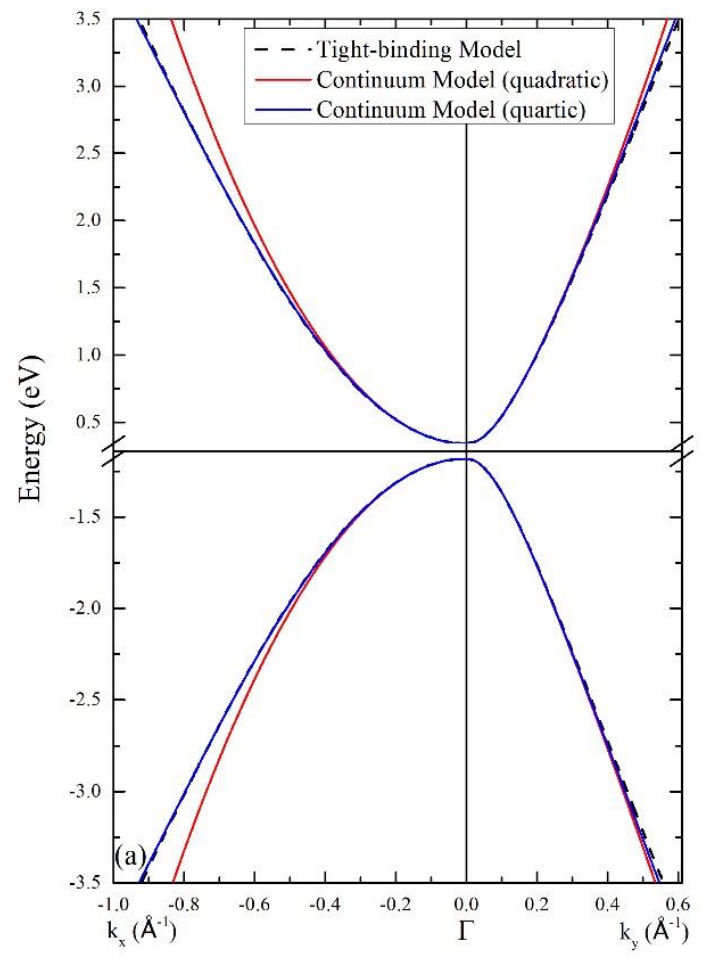

$$
\operatorname{DOS}=\int \frac{d k_{x} d k_{y}}{(2 \pi)^{2}} \delta\left(E-E_{\lambda}\right)
$$

To calculate DOS, we consider the Gaussian broadening as an approximation to the Dirac function, i.e., $\delta\left(E-E_{\lambda}\right) \approx(1 / \sigma \sqrt{\pi}) \exp [-(E-$ $\left.\left.E_{\lambda}\right)^{2} / \sigma^{2}\right]$, with $\sigma$ is the broadening parameter taken to be $\sigma=5 \mathrm{meV}$. We also present the DOS

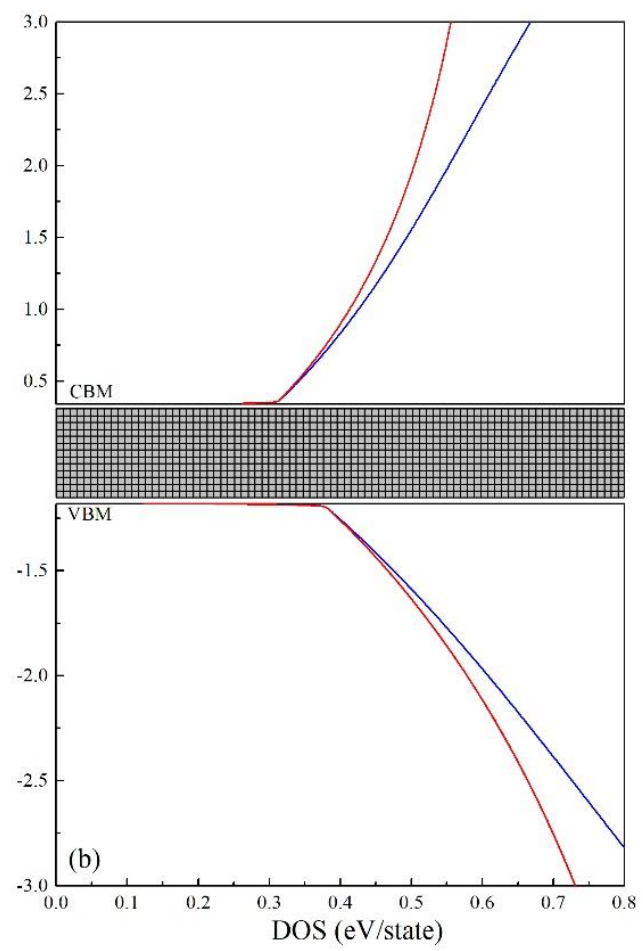

Figure 2. (a) Low energy spectrum of phosphorene for tight-binding, quadratic and quartic continuum models. (b) DOS of phosphorene for quadratic and quartic continuum models. Shaded area corresponds to band gap between CBM and VBM at $\Gamma$ point. (The tight-binding curve is generated by using tight-binding Hamiltonian in Ref. 21).

curve of phosphorene for both quadratic and quartic energy eigenvalues in Fig.2(b). As it is seen, while both model match for the low energies around energy gap, by the increasing of energies, quartic model has more contribution to the electronic states. On the other hand, Van Hove singularity at valance band of phosphorene arising from the quartic term was showed in Ref. 24 by using density functional theory scheme. In Ref. 24, the continuum model of phosphorene is extracted from the results of the density functional theory which yields a new valance band maximum (VBM) closed to the $\Gamma$ point. That saddle point around VBM is responsible from the Van Hove singularity at valance band of phosphorene. Since the quartic continuum energy eigenvalues extracted from tightbinding model do not have a new VBM or conduction band minimum (CBM), Van Hove singularity cannot be captured in this model, thus we see the smooth curves for the DOS of phosphorene in Fig.2(b). 


\section{B. Landau levels with quartic correction}

In the presence of perpendicular magnetic field, the quartic continuum Hamiltonian of phosphorene in Eq.(2) can be written as,

$$
\mathcal{H}_{0}=\left[\begin{array}{cc}
u_{0}+\bar{\eta}_{x} \pi_{x}^{2}+\bar{\eta}_{y} \pi_{y}^{2}+\bar{\tau}_{x} \pi_{x}^{4}+\bar{\gamma}_{y} \pi_{y}^{4} & \delta+\bar{\gamma}_{x} \pi_{x}^{2}+\bar{\gamma}_{y} \pi_{y}^{2}+\bar{\kappa}_{x} \pi_{x}^{4}+\bar{\kappa}_{y} \pi_{y}^{4}+i \bar{\chi} \pi_{y} \\
\delta+\bar{\gamma}_{x} \pi_{x}^{2}+\bar{\gamma}_{y} \pi_{y}^{2}+\bar{\kappa}_{x} \pi_{x}^{4}+\bar{\kappa}_{y} \pi_{y}^{4}-i \bar{\chi} \pi_{y} & u_{0}+\bar{\eta}_{x} \pi_{x}^{2}+\bar{\eta}_{y} \pi_{y}^{2}+\bar{\tau}_{x} \pi_{x}^{4}+\bar{\tau}_{y} \pi_{y}^{4}
\end{array}\right]
$$

Eq.(5) can be divided in terms of non-linear and linear terms, respectively, as

$$
\mathcal{H}_{0}^{\text {non-lin }}=\left[\begin{array}{cc}
u_{0}+\bar{\eta}_{x} \pi_{x}^{2}+\bar{\eta}_{y} \pi_{y}^{2}+\bar{\tau}_{x} \pi_{x}^{4}+\bar{\tau}_{y} \pi_{y}^{4} & \bar{\gamma}_{x} \pi_{x}^{2}+\bar{\gamma}_{y} \pi_{y}^{2}+\bar{\kappa}_{x} \pi_{x}^{4}+\bar{\kappa}_{y} \pi_{y}^{4} \\
\bar{\gamma}_{x} \pi_{x}^{2}+\bar{\gamma}_{y} \pi_{y}^{2}+\bar{\kappa}_{x} \pi_{x}^{4}+\bar{\kappa}_{y} \pi_{y}^{4} & u_{0}+\bar{\eta}_{x} \pi_{x}^{2}+\bar{\eta}_{y} \pi_{y}^{2}+\bar{\tau}_{x} \pi_{x}^{4}+\bar{\tau}_{y} \pi_{y}^{4}
\end{array}\right]
$$

and

$$
\mathcal{H}_{0}^{l i n}=\left[\begin{array}{cc}
0 & \delta+i \bar{\chi} \pi_{y} \\
\delta-i \bar{\chi} \pi_{y} & 0
\end{array}\right]
$$

where $\pi_{\mathrm{i}}$ is the $2 \mathrm{D}$ canonical momentum. In symmetric gauge, $\pi_{x}=p_{x}-(e B / 2) y$, and $\pi_{y}=$ $p_{y}+(e B / 2) x$ where $p_{i}$ is the momentum operator. It is possible to represent $p_{i}$ and $r_{i}$ in terms of the creation $b_{i}^{\dagger}$ and annihilation $b_{i}$ operators as

$$
\begin{aligned}
& p_{i}=\left(\frac{m_{\lambda i} \hbar \omega_{\lambda}}{2}\right)^{1 / 2}\left(b_{i}^{\dagger}+b_{i}\right) \\
& r_{i}=-i\left(\frac{\hbar}{2 m_{\lambda i} \omega_{\lambda}}\right)^{1 / 2}\left(b_{i}^{\dagger}-b_{i}\right), \\
& E_{n}^{n o n-l i n}=u_{0}+\left(\bar{\eta}_{x}+\lambda \bar{\gamma}_{x}\right)\left\langle n_{x} n_{y}\left|\pi_{x}^{2}\right| n_{x} n_{y}\right\rangle+\left(\bar{\eta}_{y}+\lambda \bar{\gamma}_{y}\right)\left\langle n_{x} n_{y}\left|\pi_{y}^{2}\right| n_{x} n_{y}\right\rangle \\
& +\left(\bar{\tau}_{x}+\lambda \bar{\kappa}_{x}\right)\left\langle n_{x} n_{y}\left|\pi_{x}^{4}\right| n_{x} n_{y}\right\rangle+\left(\bar{\tau}_{y}+\lambda \bar{\kappa}_{y}\right)\left\langle n_{x} n_{y}\left|\pi_{y}^{4}\right| n_{x} n_{y}\right\rangle
\end{aligned}
$$

where 


$$
\begin{aligned}
\left\langle n_{x} n_{y}\left|\pi_{x}^{2}\right| n_{x} n_{y}\right\rangle & =\frac{m_{\lambda x} \hbar \omega_{\lambda}}{2}\left(b_{x}^{\dagger}+b_{x}\right)^{2}-\left(\frac{e B}{2}\right)^{2} \frac{\hbar}{2 m_{\lambda y} \omega_{\lambda}}\left(b_{y}^{\dagger}-b_{y}\right)^{2} \\
& -i e B \sqrt{\frac{m_{\lambda x} \hbar \omega_{\lambda}}{2}} \sqrt{\frac{\hbar}{2 m_{\lambda y} \omega_{\lambda}}}\left(b_{x}^{\dagger}+b_{x}\right)\left(b_{y}^{\dagger}-b_{y}\right) \\
\left\langle n_{x} n_{y}\left|\pi_{y}^{2}\right| n_{x} n_{y}\right\rangle & =\frac{m_{\lambda y} \hbar \omega_{\lambda}}{2}\left(b_{y}^{\dagger}+b_{y}\right)^{2}-\left(\frac{e B}{2}\right)^{2} \frac{\hbar}{2 m_{\lambda x} \omega_{\lambda}}\left(b_{x}^{\dagger}-b_{x}\right)^{2} \\
& +i e B \sqrt{\frac{m_{\lambda y} \hbar \omega_{\lambda}}{2}} \sqrt{\frac{\hbar}{2 m_{\lambda x} \omega_{\lambda}}}\left(b_{y}^{\dagger}+b_{y}\right)\left(b_{x}^{\dagger}-b_{x}\right) \\
\left\langle n_{x} n_{y}\left|\pi_{x}^{4}\right| n_{x} n_{y}\right\rangle & =\left[\left(\frac{m_{\lambda x} \hbar \omega_{\lambda}}{2}\right)^{1 / 2}\left(b_{x}^{\dagger}+b_{x}\right)+(i e B / 2)\left(\frac{\hbar}{2 m_{\lambda y} \omega_{\lambda}}\right)^{1 / 2}\left(b_{y}^{\dagger}-b_{y}\right)\right]^{4} \\
\left\langle n_{x} n_{y}\left|\pi_{y}^{4}\right| n_{x} n_{y}\right\rangle & =\left[\left(\frac{m_{\lambda y} \hbar \omega_{\lambda}}{2}\right)^{1 / 2}\left(b_{y}^{\dagger}+b_{y}\right)-(i e B / 2)\left(\frac{\hbar}{2 m_{\lambda x} \omega_{\lambda}}\right)^{1 / 2}\left(b_{x}^{\dagger}-b_{x}\right)\right]^{4} .
\end{aligned}
$$

On the other hand, the linear term, $H_{0}^{\text {lin }}$ can be diagonalized by taking its square as,

$$
\left(E_{n}^{l i n}\right)^{2}=\delta^{2}+\bar{\chi}^{2}\left\langle n_{x} n_{y}\left|\pi_{y}^{2}\right| n_{x} n_{y}\right\rangle
$$

Assuming $n_{x}=n_{y}=n$ by considering the gauge independent degeneracy of Landau levels, the eigenvalues of $H_{0}^{\text {lin }}$ Hamiltonian are given by

$$
\begin{aligned}
E_{n \lambda}^{\text {quartic }} & =E_{n}^{\text {non-lin }}+E_{n}^{\text {lin }} \\
& =u_{0}+\lambda\left[\left|\left(\bar{\eta}_{x}+\lambda \bar{\gamma}_{x}\right)\right| m_{\lambda x}+\left|\left(\bar{\eta}_{y}+\lambda \bar{\gamma}_{y}\right)\right| m_{\lambda y}\right] \hbar \omega_{\lambda}\left(n+\frac{1}{2}\right) \\
& +\left[\left(\bar{\tau}_{x}+\lambda \bar{\kappa}_{x}\right) m_{\lambda x}^{2}+\left(\bar{\tau}_{y}+\lambda \bar{\kappa}_{y}\right) m_{\lambda y}^{2}\right]\left(\hbar \omega_{\lambda}\right)^{2}\left(6 n^{2}+6 n+3\right) \\
& +\lambda\left[\delta^{2}+\bar{\chi}^{2} m_{\lambda y} \hbar \omega_{\lambda}\left(n+\frac{1}{2}\right)\right]^{\frac{1}{2}} .
\end{aligned}
$$

Notice that, similar method was followed in Ref. 25 for the Landau levels of phosphorene. In the absence of quartic terms, Eq.(11) becomes

$$
\begin{aligned}
E_{n \lambda}^{\text {quadratic }} & =u_{0}+\lambda\left[\left|\left(\bar{\eta}_{x}+\lambda \bar{\gamma}_{x}\right)\right| m_{\lambda x}+\left|\left(\bar{\eta}_{y}+\lambda \bar{\gamma}_{y}\right)\right| m_{\lambda y}\right] \hbar \omega_{\lambda}\left(n+\frac{1}{2}\right) \\
& +\lambda\left[\delta^{2}+\bar{\chi}^{2} m_{\lambda y} \hbar \omega_{\lambda}\left(n+\frac{1}{2}\right)\right]^{\frac{1}{2}} .
\end{aligned}
$$

Here, the last term in Eqs.(11) and (12) can be expanded as,

$$
\delta\left[1+\left(\bar{\chi}^{2} m_{\lambda y} / \delta^{2}\right) \hbar \omega_{\lambda}(n+1 / 2)\right]^{1 / 2} \approx\left[\delta+\left(\bar{\chi}^{2} m_{\lambda y} / 2 \delta\right) \hbar \omega_{\lambda}(n+1 / 2)\right]
$$

and finally we arrive at 


$$
\begin{aligned}
E_{n \lambda}^{\text {quadratic }} & =\left(u_{0}+\lambda \delta\right)+\lambda \hbar \omega_{\lambda}\left(n+\frac{1}{2}\right) \\
E_{n \lambda}^{\text {quartic }} & =\left(u_{0}+\lambda \delta\right)+\lambda \hbar \omega_{\lambda}\left(n+\frac{1}{2}\right)+\xi_{\lambda}\left(\hbar \omega_{\lambda}\right)^{2}\left(6 n^{2}+6 n+3\right) .
\end{aligned}
$$

Here, $\xi_{\lambda}$ takes 0.014 for electrons $(\lambda=1)$ and 0.018 for holes $(\lambda=-1)$. Square root and linear energy expressions in Eqs.(12) and (13), are plotted in Fig.3(a). It is clear that below the $B \approx$ $30 \mathrm{~T}$, both expression of quartic model are perfectly matched for the whole values of Landau level index, $n$. According the energy expression in Eqs.(3) and (4) in the presence of magnetic field, linear term is less effective on Landau levels of phosphorene. Similar behavior of quadratic continuum energy was also discussed in Refs. 21 and 25. It can be also concluded that electronhole asymmetry is present at Landau level spectrum due to the anisotropic structure of phosphorene having different effective masses of charge carriers. As it can be followed from the second term in Eq.(13), the quartic terms yield non-linearity at the Landau level spectrum with the magnetic field. Even the non-linearity has small effect on Landau levels of phosphorene, it is demonstrated in an experimental study for few-layer black phosphorus. ${ }^{26}$ The relative energies $\Delta E_{n}^{\lambda}=E_{n \lambda}^{\text {quadratic }}-E_{n \lambda}^{\text {quartic }}$ of Landau levels with magnetic field are plotted in Figs.3(b) and (c). It is obvious that non-linearity can be seen clearly from the behavior of curves for the different Landau level index. Even the contribution of non-linear term arising from the quartic model has negligible effect on lower
Landau levels, the behavior of the Landau levels shows different trend according to the Landau level index, $n$. While the energies of quadratic model are decreased with quartic terms for the lower Landau level index, they are enhanced for the higher index. In other words, it can be easily claimed that by inclusion of $k^{4}$ terms the energy of ground state (ground and first-excited states) is increased for electron (holes), on the other part, quartic terms reduce the energy of the Landau levels for the excited states (excited states above $n=1$ ) for electron (hole). Moreover, there are some critical magnetic field values where both energy expressions of quadratic and quartic model are equal. Under these critical values, quartic terms decrease the energy of whole Landau levels insignificantly. It is also clear from the Figs.3 (b) and (c) that these critical values are decreased with the increasing values of Landau level index. Even trend of curves is similar, difference between electron and hole state arises from their effective masses which is based on the anisotropy of phosphorene. If we compare the scales of relative energies belong to electron and hole in Figs.3(b) and (c), quartic terms also induce asymmetry between electron and hole states due to the effective mass dependent contribution of quartic term (see last term in Eq.(13)). 


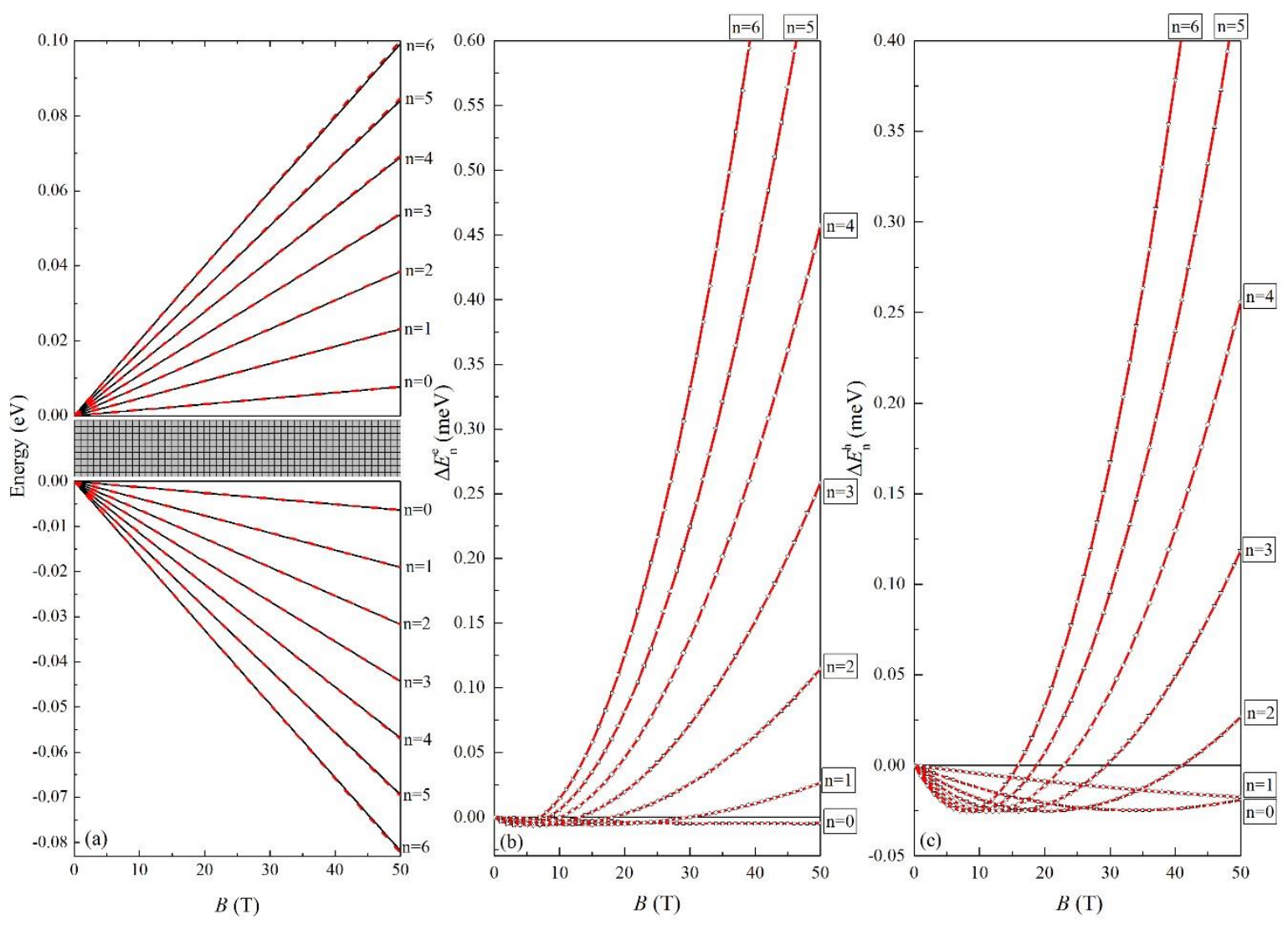

Figure 3. (a) Landau levels of MBP, red dashed line corresponds to a linear expression in Eq. (13), black line corresponds to Eq. (12), relative energies $\left(\Delta E_{n}^{\lambda}\right)$ for (b) electrons and (c) holes.

\section{CONCLUSIONS}

In summary, we extract a new continuum model based on quartic wave-vectors $\left(k^{4}\right)$ from the tight-binding model of phosphorene. We examine the energy eigenvalues of quartic Hamiltonian in the presence and absence of magnetic field. We see that quartic model brings better description according to the well-known quadratic model for the behavior of charge carriers in phosphorene. In the absence of magnetic field, quartic model increases the range of energy where eigenvalues of continuum model match with tight-binding results. We also see from the DOS curve, quadratic model underestimates the contribution arising from the electronic states. In the presence of perpendicular magnetic field to phosphorene plane, quartic terms yield non-linearity at the energy spectrum of the system. We also analyze the Landau levels of phosphorene in the framework of quartic continuum model. We conclude that quartic terms in continuum model have insignificant effect on lower $(n=0$ and 1$)$ Landau levels of phosphorene. However, they have non-negligible contribution to the higher Landau levels of phosphorene at the order of $\sim m e V$. Similarly, the extension of continuum model in graphene was achieved by the trigonal warp effect which increases the energy regime of Dirac Hamiltonian to bring better description for the dynamics of graphene charge carriers $[2,18]$. As a conclusion, it can be maintained that the presented continuum model in here will lead better understanding for the electronic and transport properties of phosphorene in the framework of $k \cdot p$ theory. 


\section{REFERENCES}

[1]. Novoselov, K. S., Geim, A. K., Morozov, S. V., Jiang, D., Zhang, Y., Dubonos, S. V., Grigorieva, I. V., Firsov, A. A. 2004. Science 306: 666-669.

[2]. Katsnelson, Mikhail I. Graphene: carbon in two dimensions, 1st ed., Cambridge University Press, 2012.

[3]. Joshua, B. S., Hagaman, D., Hai-Feng, J. 2016. Nanotechnology 27: 215602.

[4]. Favron, A., Gaufrès, E., Fossard, F., Phaneuf-LHeureux, A. L., Tang, N. Y. W., Lévesque, P. L., Loiseau, A., Leonelli, R., Francoeur, S., Martel, R., 2015. Nat. Mater. 14: 826-832.

[5]. Brent, Jack R., Savjani, Nicky, Lewis, Edward A., Haigh, Sarah J., Lewis, David J., O'Brien, Paul 2014. Chem. Commun. 50: 13338-13341.

[6]. Liu, Han, Neal, Adam T., Zhu, Zhen, Luo, Zhe, Xu, Xianfan, Tomnek, D., Ye, Peide D. 2014. ACS Nano 8: 4033-4041.

[7]. Li, L., Yu, Y., Ye, G. J., Ge, Q., Ou, X., Wu, H., Feng, D., Chen, X. H., Zhang, Y. 2014. Nat. Nanotech. 9: 372.

[8]. Koenig, S. P., Doganov, R. A., Schmidt, H., Castro Neto, A. H., Ö zyilmaz, B. 2014. Appl. Phys. Lett. 104: 103106.

[9]. Tran, V., Soklaski, R., Liang, Y., Yang, L. 2014. Phys. Rev. B 89: 235319.

[10]. Qiao, Jingsi, Kong, Xianghua, Hu, ZhiXin, Yang, Feng, Ji, Wei 2014. Nat. Commun. 5: 4475.

[11]. Xia, F., Wang, H., Jia, Y. 2014. Nat. Commun. 5: 4458.
[12]. Wang, X., Jones, A. M., Seyler, K. L., Tran, V., Jia, Y., Zhao, H., Wang, H., Yang, L., Xu, X., Xia, F. 2015. Nat. Nanotech. 10: 517.

[13]. Rudenko, A. N., Yuan, Shengjun, Katsnelson, M. I. 2015. Phys. Rev. B 92: 085419.

[14]. Rudenko, A. N., Katsnelson, M. I. 2014. Phys. Rev. B 89: 201408.

[15]. Jain, Ankit, McGaughey, Alan J. H. 2015. Scientific Reports 5: 8501.

[16]. Semenoff, Gordon W. 1984. Phys. Rev. Lett. 53: 2449-2452.

[17]. Katsnelson, M.I., Novoselov, K.S., Geim, A.K. 2006. Nature Physics 2: 620-625.

[18]. Ajiki, H., Ando, T. 1996. J. Phys. Soc. Jpn. 65: 505-514.

[19]. Rakyta, P., Kormányos, A., Cserti, J. 2010. Phys. Rev. B 82: 113405.

[20]. Azizi, J., Phirouznia, A., Hasanirokh, K. 2015. Physica E 68: 28-32.

[21]. Pereira, J. M., Katsnelson, M. I. 2015. Phys. Rev. B 92: 075437.

[22]. Zhou, X. Y., Zhang, R., Sun, J. P., Zou, Y. L., Zhang, D., Lou, W. K., Cheng, F., Zhou, G. H., Zhai, F., Chang, Kai 2015. Scientific Reports 5: 12295.

[23]. Ezawa, M. 2014. New J. Phys. 16: 115004.

[24]. Ziletti, A., Huang, S. M., Coker, D. F., Lin, H. 2015. Phys. Rev. B 92: 085423.

[25]. Mogulkoc, A., Modarresi, M., Rudenko, A. N. 2017. Phys. Rev. B 96: 085434.

[26]. Long, G., Maryenko, D., Pezzini, S., Xu, S., Wu, Z., Han, T., Lin, J., Wang, Y., An, L., Cai, Y., Zeitler, U., Wang, N. 2017. arXiv preprint arXiv:1703.05177. 